\title{
Development of an Attenuated Total Reflection Based Fiber-Optic Sensor for Real-time Sensing of Biofilm Formation
}

\author{
Tatsuya OriI,* Takuya OKazaKI,* Noriko Hata,* Kazuharu Sugawara, ${ }^{* *}$ Faidz A. Rahman, ${ }^{* * *}$ \\ and Hideki KurAMITZ ${ }^{* \dagger}$
}

\author{
*Department of Environmental Biology and Chemistry, Graduate School of Science and Engineering for \\ Research, University of Toyama, 3190 Gofuku, Toyama 930-8555, Japan \\ **Maebashi Institute of Technology, Maebashi, Gunma 371-0816, Japan \\ ***Department of Electrical and Electronic Engineering, Lee Kong Chian Faculty of Engineering and Science, \\ Universiti Tunku Abdul Rahman, Jalan Sungai Long, Bandar Sungai Long, Cheras, 43000 Kajang, \\ Selangor, Malaysia
}

\begin{abstract}
A fiber-optic sensor capable of real-time monitoring of biofilm formation in water was developed. The sensor can be easily fabricated by removing the cladding of a multimode fiber optic to expose the core. The sensing action is based on the penetration of an evanescent wave through a biofilm formed on the surface of the exposed fiber core during total internal reflection. The proposed setup can be used to analyze the transmittance response over a wide wavelength range using a white-light source and a spectroscopy detector. The change in transmittance with respect to the biofilm formation on the fiber core surface was observed. The findings from this study showed that the sensor detection had better sensitivity at near-infrared wavelengths than at visible-light wavelengths. Moreover, the sensitivity of this sensor could be controlled by surface modifications of the core surface through electrostatic interactions, involving a silane coupling layer, polyanions, and polycations. The developed sensor was successfully applied to evaluating of the effectiveness of a commercial biofilm inhibitor.
\end{abstract}

Keywords Biofilm, fiber-optic sensor, real-time sensing, electrostatic charge, biofilm inhibitor

(Received January 18, 2017; Accepted March 3, 2017; Published August 10, 2017)

\section{Introduction}

A biofilm is a community of surface-associated microbial cells enclosed within an exopolymer matrix, which adheres to an inert or living surface. The formation of biofilms in a water system can be detrimental to human health, and is the root of many recalcitrant infections and diseases. ${ }^{1,2}$ Biofilm formation can also affect industrial production by reducing the flow rate of water and the heat-exchange efficiency in the heat exchange system. ${ }^{3,4}$ To prevent this problem, numerous studies have been conducted on inhibitors of bacterial biofilm formation; some such inhibitors are being used in industrial water process systems. ${ }^{5}$ On the other hand, biofilms are beneficially utilized in various technologies, such as water treatment, biohydrogen production, bioleaching, and microbial electrocatalysis. ${ }^{6-9}$ Therefore, it is important to monitor biofilm formation in real time and to evaluate the effectiveness of inhibitors in industrial water systems. Furthermore, continuous monitoring of the growth of beneficial biofilms is vital in environmental technologies.

Various techniques for monitoring biofilm formation have been reported in the past three decades. ${ }^{10}$ Microscopy imaging is one of the techniques that provide information concerning the

† To whom correspondence should be addressed.

E-mail: kuramitz@sci.u-toyama.ac.jp physical structure, cell size, shape, and chemical properties. However, it is a labor-intensive method, and cannot be implemented for real-time monitoring. In order to overcome these obstacles, techniques such as localized surface plasmon resonance spectroscopy, ellipsometry, voltammetry, photoacoustic spectroscopy, and fluorescence sensing have been suggested over the years, but they have limited application and the obstacles still exist. ${ }^{11-15}$

Evanescent field spectroscopy is an extension of attenuated total reflection (ATR) spectroscopy, an established spectroscopic technique. This technique is based on the penetration of an evanescent wave through the sensing sample during total internal reflection. The use of fiber optics in the detection of chemicals and bioanalytes has recently been increasing. ${ }^{16-20}$ The concept of evanescent field spectroscopy has been applied to the fiberoptic ATR technique, where the exposed core of a fiber acts as a sensing waveguide. The fiber-optic ATR method has a number of advantages in terms of miniaturization, low cost, ease of handling, and real-time remote monitoring. Apart from its ability to sense multiple chemicals, the fiber-optic evanescent wave sensor can be operated in broad wavelength regions, such as ultraviolet (UV), visible (Vis), near infrared (NIR), and midinfrared. ${ }^{21-29}$

Several groups have studied the fiber-optic method for monitoring biofilm formation by using an exposed fiber-optic core. ${ }^{4,10,30}$ These methods were based on the principle of evanescent field attenuation by biofilm formation. In our 


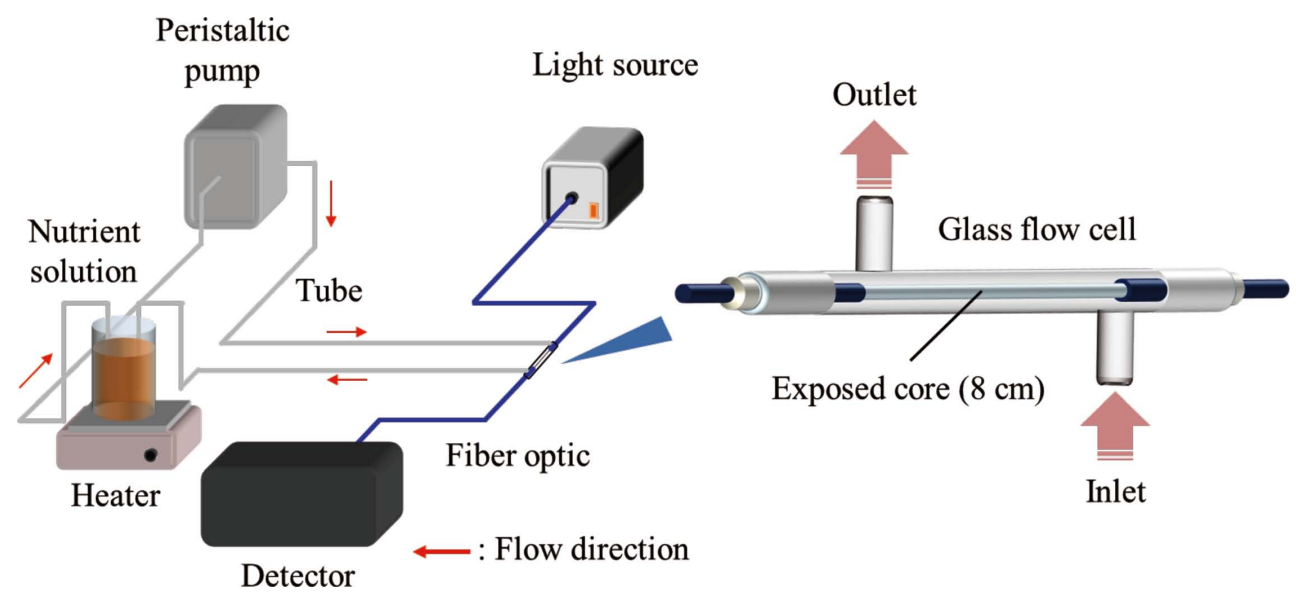

Fig. 1 Scheme of the experimental setup of biofilm sensing.

previous work, we proposed an optical fiber sensor for $\mathrm{CaCO}_{3}$ and silica scale formation in geothermal water using an exposed fiber-optic core and a spectroscopy detector. ${ }^{31,32}$ The sensor detection principle is based on the optical transmittance over a wide wavelength range, which is affected by the high refractive index of $\mathrm{CaCO}_{3}$ or silica scale formed on the exposed core. In addition, we proposed a spectroelectrochemical fiber-optic sensor, whose sensitivity for model analytes can be controlled by electrostatic modification using self-assembled monolayer (SAM) formation on the surface of the fiber-optic core. ${ }^{33}$

In the present study, an optimized fiber-optic sensor was developed for monitoring biofilm formation in a nutrient solution. The optical response was investigated based on the optimization of a selected wavelength using a white-light source and a spectroscopy detector in the spectral Vis-NIR range. The sensitivity control technique by the abovementioned SAM modification of the fiber sensor was applied for monitoring biofilm formation. The amount of biofilm formed was analyzed to understand changes in the sensor responses. The fiber-optic sensing of biofilm formation proposed in this study was investigated in a nutrient solution containing a biofilm inhibitor, and the effectiveness of the inhibitor was evaluated.

\section{Experimental}

\section{Chemical and materials}

Yeast extract was obtained from Becton, Dickinson and Company (USA). Glucose, crystal violet, acetone, and sodium hydroxide were purchased from Wako Pure Chemical Industries (Japan). A biofilm inhibiter was obtained from Gospel Toshin Ltd. (Japan). Poly(allylaminehydrochloride) (PAH) and poly\{1-[4-((3-carboxy-4-hydroxyphenyl) azo)-benzenesulfonamido]1,2-ethanediyl, sodium salt $\}$ (PCBS) were obtained from SigmaAldrich (USA). (3-Aminopropyl) triethoxysilane (APTES) was purchased from Tokyo Chemical Industry (Japan). All chemicals were used as received without further purification, and Milli-Q water was used in solution preparation.

A multimode plastic-clad silica fiber (FT200EMT, refractive index: 1.44) with a $200 \mu \mathrm{m}$-step index for the core diameter was purchased from ThorLabs (USA). The cladding was removed $8 \mathrm{~cm}$ length from the center using acetone, and the exposed fiber core was used as the sensing region.

\section{Preparation of a self-assembled monolayer on the fiber}

For PAH and PCBS modifications, an APTES-immobilized fiber was prepared using a silane coupling reaction. The exposed core was activated in preparation for silanization by soaking in $1.0 \mathrm{M}$ sodium hydroxide for $1 \mathrm{~h}$, followed by rinsing with Milli-Q water. The core surface was subsequently dipped in acetone containing $2 \%(\mathrm{v} / \mathrm{v})$ APTES for $1 \mathrm{~min}$ at room temperature to accomplish silanization. After silanization, a SAM was applied onto the core. In our experiments, PAH and PCBS were chosen as the polycation and polyanion, respectively, for the SAM. We applied the modified ionic SAM onto the fiber-optic-immobilized APTES, as previously reported. ${ }^{34,35}$ After rinsing with Milli-Q water, a PCBS layer was formed on the APTES layer by immersing the fiber-optic-immobilized APTES in a solution containing $10 \mathrm{mM}$ PCBS for $15 \mathrm{~min}$ at room temperature. Then, the core-immobilized PCBS/APTES was soaked in $10 \mathrm{mM}$ PAH solution for $15 \mathrm{~min}^{35-37}$

\section{Sensing of biofilm formation}

An ELI-050J-OPT3077 (Mitsubishi rayon, Japan) white-light source device attached to a JCR12V-50WGAL (Ushio, Japan) halogen lamp was used to perform light coupling between the white-light source and an SA-100VRD Vis-NIR (Lambda vision, Japan) spectroscopy detector through the fiber sensor. The fiber sensor was placed into a flow cell consisting of a 3.0-mm-diameter glass tube connected to a peristaltic pump and a nutrient solution containing $1 \%$ yeast extract and glucose in a reservoir, as shown Fig. 1. The nutrient solution was maintained at $40^{\circ} \mathrm{C}$ using a temperature controller placed under the reservoir, and was flown through the glass cell at a volumetric flow rate of $120 \mathrm{~mL} / \mathrm{h}$ using the peristaltic pump. Transmittance values were acquired by recording the light intensity through the fiber sensor when exposed to the nutrient solution $\left(I_{0}\right)$ and after exposure to biofilm formation as time proceeded $(I)$. The transmittance of the fiber sensor was defined as $T(\%)=\left(I / I_{0}\right) \times 100$. The transmittance spectrum was measured continuously over the $600-1600 \mathrm{~nm}$ wavelength region.

\section{Measurement of biofilm adhered to the sensor}

For measuring of the amount of biofilm formed, the exposed fiber core was placed into a nutrient solution maintained at $40^{\circ} \mathrm{C}$. After a certain time, the fiber was removed from the solution, and biofilm adhered to the fiber core was dyed with $0.1 \%$ crystal violet solution. ${ }^{38}$ The resulting surface of the fiber core was observed by optical microscopy with a CX41 

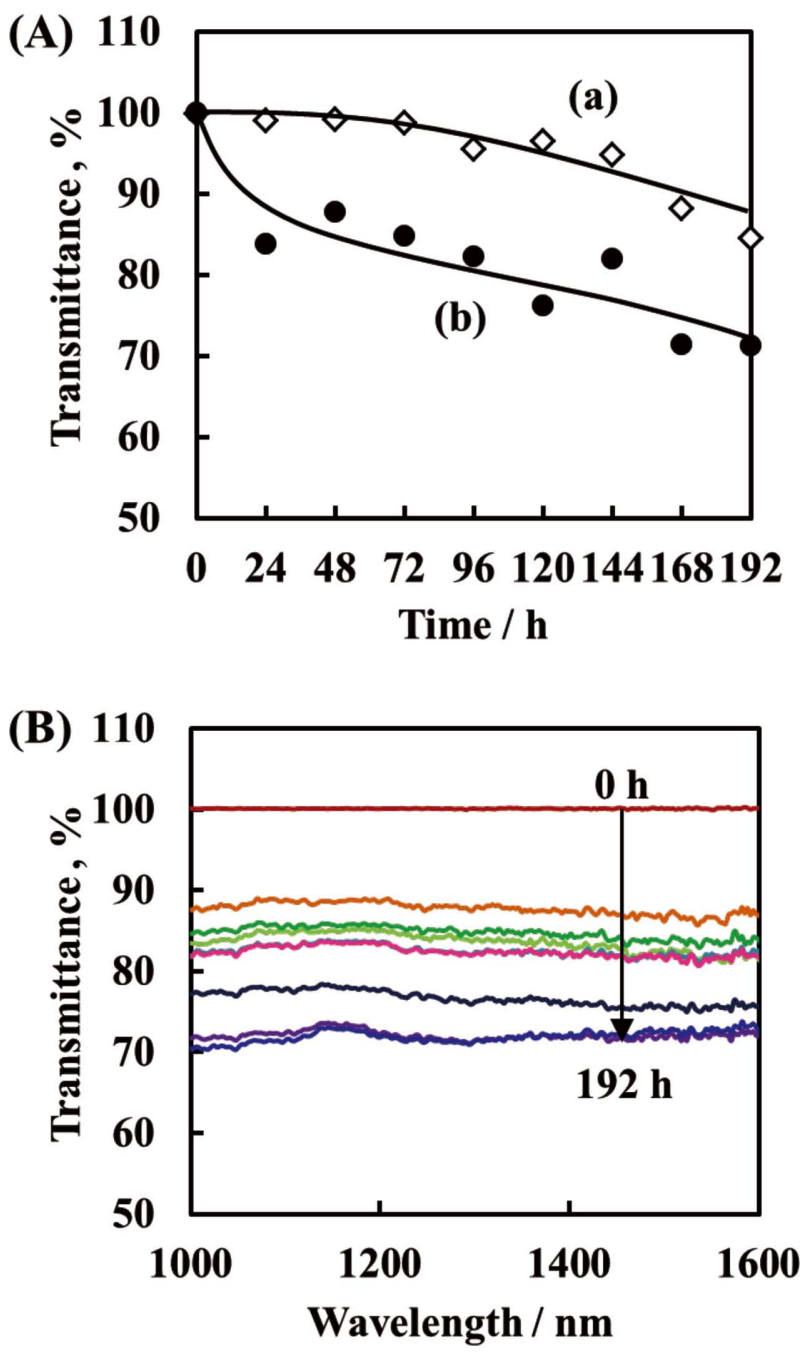

Fig. 2 (A) Transmittance responses monitored by a fiber-optic sensor at wavelengths $700 \mathrm{~nm}$ (a) and $1300 \mathrm{~nm}$ (b) as a function of time after immersing the sensor in a nutrient solution containing $1 \%$ yeast extract and glucose. (B) Spectral changes from 1000 to $1700 \mathrm{~nm}$ under the same condition. Curves from top to bottom represent the different times of $0,24,48,72,96,120,144,168$, and $192 \mathrm{~h}$ after immersion in the solution.

microscope (Olympus, Japan). After the observation, the dye was eluted with $3 \mathrm{~mL}$ of $95 \%$ ethanol. The absorbance of this solution was measured at $590 \mathrm{~nm}$ (UV-2550, Shimadzu, Japan).

\section{Results and Discussion}

\section{Transmittance at various wavelengths}

The transmittance response of the fiber sensor was investigated at several wavelengths ranging from 600 to $1600 \mathrm{~nm}$. The response, shown in Fig. 2(A), indicates that the transmittance decreased to a constant value over time. Broad lines were observed in the range 1000 - $1600 \mathrm{~nm}$ (Fig. 2(B)). Furthermore, the transmittance response showed better sensitivity in the NIR region than in the Vis region. The higher sensitivity in the NIR region might be related to the penetration depth of evanescent waves into the biofilm. The evanescent wave is generated between the fiber core and the biofilm, which has a lower refractive index than that of the core, when the light traveling

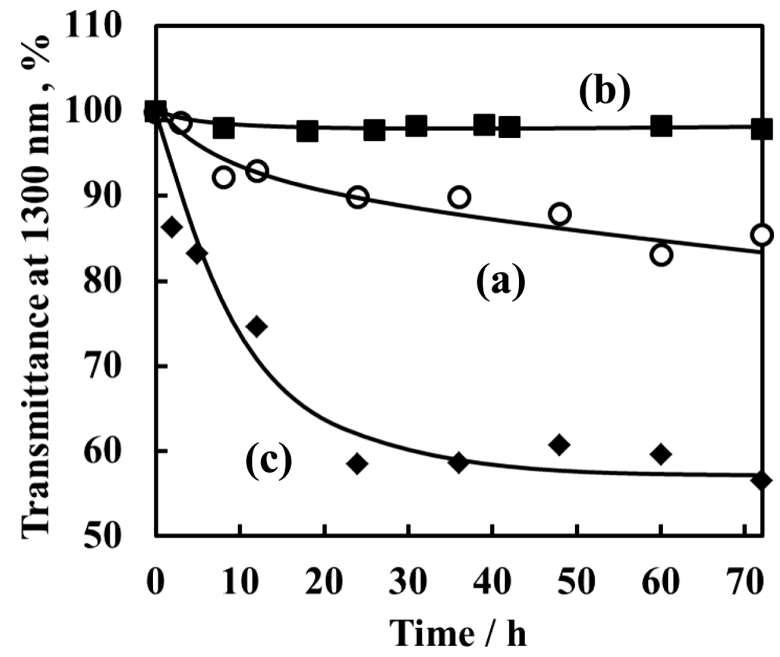

Fig. 3 Transmittance responses to biofilm formation obtained from a bare fiber sensor (a), a PCBS-modified fiber sensor (b), and a PAHmodified fiber sensor (c) at $1300 \mathrm{~nm}$ as a function of time after immersing the nutrient solution.

within the fiber undergoes total internal reflection. ${ }^{4}$ This evanescent wave is then absorbed by the biofilm instead of getting reflected into the fiber core. The penetration depth, $d_{\mathrm{p}}$, of the evanescent wave is directly proportional to the wavelength of the probing radiation, which is given by

$$
d_{\mathrm{p}}=\frac{\lambda}{\sqrt{\sin ^{2} \phi-\left(\frac{n_{2}}{n_{1}}\right)^{2}}},
$$

where $\lambda$ is the wavelength of light; $\phi$ is the angle of incidence; and $n_{1}$ and $n_{2}$ are the refractive indices of the core and the surrounding, respectively. ${ }^{39}$ Therefore, it can be said that the sensor has a higher sensitivity for biofilm formation in the NIR region than in the Vis region because of the longer effective path length, as shown in Fig. 2(A). There were no significant differences observed in the sensor response at the NIR region for the wavelength ranging from 1000 to $1600 \mathrm{~nm}$. Therefore, $1300 \mathrm{~nm}$ was chosen for monitoring the biofilm formation. After $72 \mathrm{~h}$ of monitoring, it was then clarified that the sensor response at this wavelength was not influenced by the solution's turbidity, which increased parallelly with the biofilm formation.

\section{Effect of electrostatic charge of the sensor surface}

The effect of the electrostatic charge of the sensor surface was examined by using a SAM, since a SAM can be used to change the electrostatic charge on the surface of materials. The optical fiber sensing of a biofilm was demonstrated using a SAM coated on the fiber surface. Figure 3 shows transmittance curves for biofilm deposition on a bare fiber sensor, a PCBS-coated fiber sensor, and a PAH-coated fiber sensor. The bare fiber surface was negatively charged due to hydroxyl groups on the quartz surface; the surfaces of the PCBS- and PAH-coated fibers were charged negatively and positively, respectively. The transmittance response obtained using the PAH-coated fiber, which was positively charged, drastically decreased to about $60 \%$ within $30 \mathrm{~h}$. A similar amount of decrement in the sensor response was observed at the NIR region of 1000 to $1600 \mathrm{~nm}$ wavelength. For the bare and PCBS-coated fibers, which were negatively charged, the transmittance change was much smaller than that of the PAH-coated fiber. This difference is due to the 

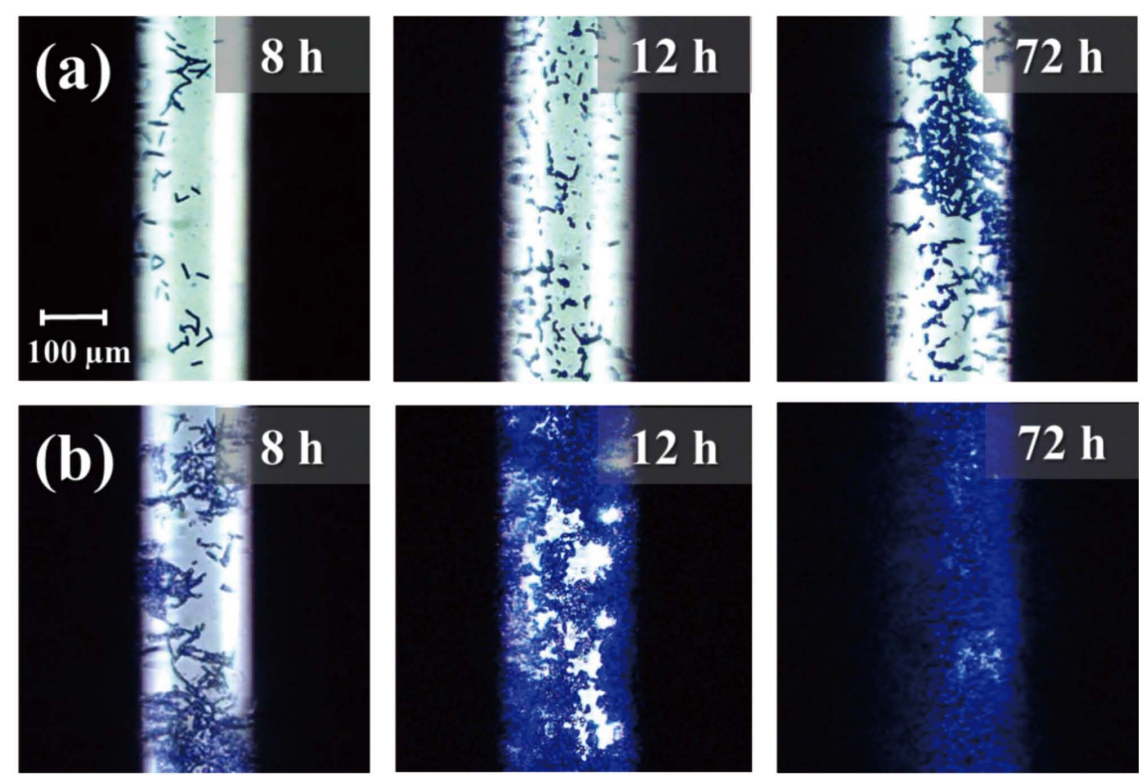

Fig. 4 Optical microscopy images of the biofilm formed on the surface of a bare fiber core (a) and a PAH-coated fiber core (b).

electrostatic charge between the surface of the biofilm and the fiber core. Matsumoto et al. suggested that biofilm adhesion to a fibrous support material, such as a carbon fiber for wastewater treatment, could be explained by the Derjaguin-LandauVerwey-Overbeek theory, which describes the interaction between the charged surface of biofilms and the support material. ${ }^{6}$ In our experiments, the bacterial biofilm has a negatively charged cell surface. The higher sensitivity of the PAH-coated fiber sensor than that of the bare and PCBS-coated fiber sensors can be attributed to the electrostatic interaction between the positively charged surface of the PAH-coated fiber sensor and the negatively charged surface of the biofilm. The difference in the transmittance change of the bare fiber and PCBS-coated fiber sensors is likely caused by the difference in the surface charge density of glass and PCBS. These results show that the sensitivity and working time of the fiber sensor for monitoring biofilms can be controlled by controlling the electrostatic charge of the exposed core surface.

\section{Fiber-optic sensor characterization of biofilm formation}

Figure 4(a) shows optical microscopy images of a biofilm dyed with crystal violet that adhered to the surface of a bare fiber core. The dyed biofilm was clearly observed on the fiber surface, and the amount of biofilm formed increased over time. On the other hand, the amount of biofilm adhered to a PAHcoated fiber was larger than that adhered to a bare fiber, as shown in Fig. 4(b). This result agreed with that of fiber-optic sensing with bare and PAH-coated sensors, as indicated in Fig. 3.

Figure 5 shows the transmittance change in the crystal violet solution extracted from dye-loaded biofilm formed on the PAHcoated fiber core. The transmittance decreased with time and attained a constant value. The tendency of the transmittance change was similar to that of the transmittance change monitored by the fiber sensor. Therefore, it is confirmed that the sensor can measure the amount of biofilm formed on the fiber core.

\section{Application for evaluation of a biofilm inhibitor}

An application of the fiber sensor for evaluating the

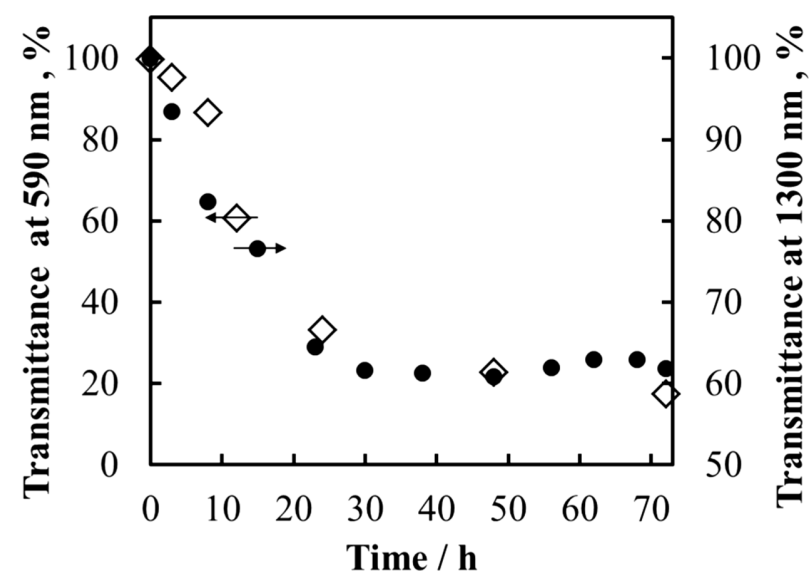

Fig. 5 Transmittance response of the fiber sensor with a PAH-coated fiber core at a wavelength of $1300 \mathrm{~nm}$ along with the $590 \mathrm{~nm}$ transmittance of crystal violet solution extracted from a dye-loaded biofilm.

effectiveness of a biofilm inhibitor was demonstrated. The biofilm inhibitor consisted of an organic nitrogen-based compound. Nutrient water containing various concentrations of the inhibitor was tested by a PAH-coated fiber sensor. The transmittance responses of the sensor at $1300 \mathrm{~nm}$ for various concentrations of the biofilm inhibitor are shown in Fig. 6. For inhibitor concentrations of 1 and $10 \mathrm{ppm}$, the transmittance changed by about $30 \%$ and $15 \%$, respectively, of the initial value within $60 \mathrm{~h}$. For an inhibitor concentration of $100 \mathrm{ppm}$, which is the manufacturer's recommended concentration, the transmittance did not change within $72 \mathrm{~h}$. It is clear that increasing the concentration of the biofilm inhibitor decreases the change in the fiber transmittance caused by biofilm formation. This observation indicates that this proposed fiber sensor can be used to obtain the optimal concentration of a biofilm inhibitor within a short time. 


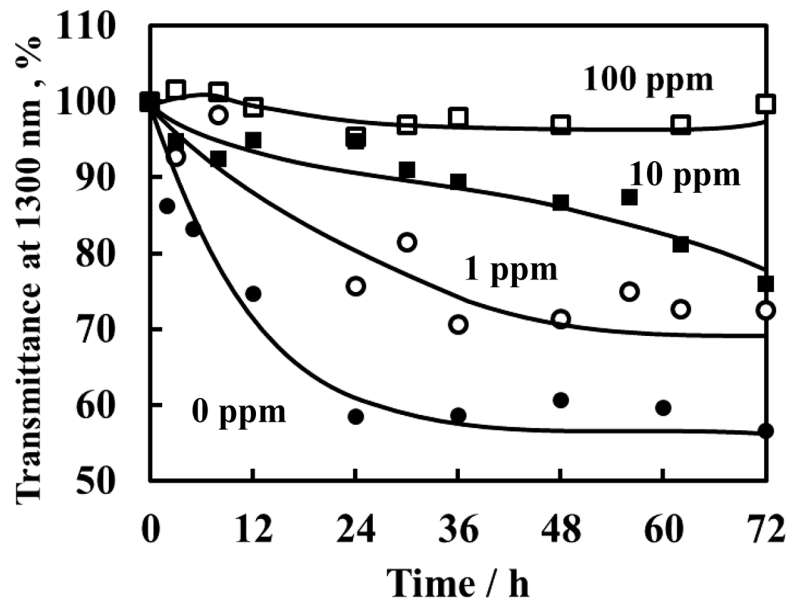

Fig. 6 Effect of the biofilm inhibitor on the transmittance response monitored by a fiber sensor at $1300 \mathrm{~nm}$ as a function of time after immersion in a nutrient solution. The curves, from top to bottom, represent the different inhibitor concentrations of $100,10,1$, and 0 ppm.

\section{Conclusions}

The experimental results suggest that the proposed sensor can be effectively used for monitoring biofilm formation. The advantages of the proposed sensor include real-time remote monitoring, small size, ease of handling, and low cost. The findings from this study showed that the detection in the NIR region has better sensitivity for biofilm formation that in the Vis region. Furthermore, the electrostatic charge on the surface of fiber core can be controlled by coating a SAM to control the sensitivity and working time of the fiber sensor for monitoring biofilms. The transmittance of the fiber sensor corresponds to the amount of biofilm formed on the fiber core. We demonstrated that the proposed sensor can be used for evaluating the effectiveness of a biofilm inhibitor.

\section{Acknowledgements}

This work was supported by JSPS KAKENHI Grant Number JP16H02976.

\section{References}

1. J. W. Costerton, P. S. Stewart, and E. P. Greenberg, Science, 1999, 284, 1318.

2. K. Lewis, Antimicrob. Agents Chemother, 2001, 45, 999.

3. Y. L. Lau and D. Liu, Water Res., 1993, 27, 335

4. R. Philip-Chandy, P. J. Scully, P. Eldridge, H. J. Kadim, M. G. Grapin, M. G. Jonca, M. G. D’Ambrosio, and F. Colin, IEEE J. Quantum Electron., 2000, 6, 764.

5. N. Rabin, Y. Zheng, C. Opoku-Temeng, Y. Du, E. Bonsu, and H. O. Sintim, Future Med. Chem., 2015, 7, 647.

6. S. Matsumoto, A. Ohtaki, and K. Hori, Environ. Sci. Technol., 2012, 46, 10175.

7. Q. Liao, N. B. Zhong, X. Zhu, R. Chen, Y. Z. Wang, and D. J. Lee, Int. J. Hydrog. Energy, 2013, 38, 15730.
8. S. Bellenberg, M. Díaz, N. Noël, W. Sand, A. Poetsch, N. Guiliani, and M. Vera, Res. Microbiol., 2014, 165, 773.

9. X. W. Liu, Y. X. Huang, X. F. Sun, G. P. Sheng, F. Zhao, S. G. Wang, and H. Q. Yu, Appl. Mater. Interfaces, 2014, 6, 8158 .

10. N. Zhong, Q. Liao, X. Zhu, and R. Chen, Anal. Chem., 2014, 86, 3994.

11. P. Pallavicini, A. Donà, A. Taglietti, P. Minzioni, M. Patrini, G. Dacarro, G. Chirico, L. Sironi, N. Bloise, L. Visai, and L. Scarabelli, Chem. Commun., 2014, 50, 1969.

12. B. Wang, J. Wang, D. Li, K. Ren, and J. Ji, Appl. Surf. Sci., 2012, 258, 7801 .

13. E. Marsili, J. B. Rollefson, D. B. Baron, R. M. Hozalski, and D. R. Bond, Appl. Environ. Microbiol., 2008, 74, 7329.

14. T. Schmid, U. Panne, J. Adams, and R. Niessner, Water Res., 2004, 38, 1189.

15. G. Wolf, J. G. Crespo, and M. A. M. Reis, Environ. Sci. Biotechnol., 2002, 1, 227.

16. O. S. Wolfbeis, Anal. Chem., 2002, 74, 2663.

17. X. Wang and O. S. Wolfbeis, Anal. Chem., 2013, 85, 487.

18. C. McDonagh, C. S. Burke, and B. D. MacCraith, Anal. Chem., 2016, 88, 203.

19. X. Wang and O. S. Wolfbeis, Chem. Rev., 2008, 108, 400.

20. E. Udd and W. B. Spillman Jr., "Fiber Optic Sensors: An Introduction for Engineers and Scientists", 2011, 2nd ed., John Wiley \& Sons, Canada.

21. R. A. Potyrailo, S. E. Hobbs, and G. M. Hieftje, Anal. Chem., 1998, 15, 1639.

22. R. Bharadwaj, V. V. R. Sai, K. Thakare, A. Dhawangale, T. Kundu, and S. Titus, Biosens. Bioelectron., 2011, 26, 3367.

23. M. D. DeGrandpre and L. W. Burgess, Anal. Chem., 1988, 60, 2582.

24. T. Yeh, C. Chu, and Y. Lo, Sens. Actuators, B, 2006, 119, 701.

25. S. Dong, M. Luo, G. Peng, and W. Cheng, Sens. Actuators, $B, \mathbf{2 0 0 8}, 129,94$.

26. Y. Xiong, D. Zhu, C. Duan, J. Wang, and Y. Guan, Anal. Bioanal. Chem., 2010, 396, 943.

27. A. B. Pillai, B. Varghese, and K. N. Madhusoodanan, Environ. Sci. Technol., 2012, 46, 404.

28. D. S. Blair, Anal. Chem., 1997, 69, 2238.

29. D. S. Blair, L. W. Burgess, and A. M. Brodsky, Appl. Spectrosc., 1995, 49, 1636.

30. M. Fischer, M. Wahl, and G. Friedrichs, Biosens. Bioelectron., 2012, 33, 172.

31. T. Okazaki, K. Imai, S. Y. Tan, Y. T. Yong, F. A. Rahman, N. Hata, S. Taguchi, A. Ueda, and H. Kuramitz, Anal. Sci., 2015, 31, 177.

32. T. Okazaki, T. Orii, A. Ueda, A. Ozawa, and H. Kuramitz, Sci. Rep., 2017, in press.

33. K. Imai, T. Okazaki, N. Hata, S. Taguchi, K. Sugawara, and H. Kuramitz, Anal. Chem., 2015, 87, 2375.

34. J. E. Park, T. Momma, and T. Osaka, Electrochim. Acta, 2007, 52, 5914.

35. G. Decher, Science, 1997, 29, 1232.

36. Z. Wang, J. R. Heflin, R. H. Stolen, and S. Ramachandran, Appl. Phys. Lett., 2005, 86, 223104-1-3.

37. Q. Li, Y. Qian, Y. Yu, G. Wu, Z. Sui, and H. Wang, Opt. Commun., 2009, 282, 2446.

38. S. Favre-Bonté, T. Köhler, and C. V. Delden, J. Antimicrob. Chemother, 2003, 52, 598.

39. B. D. Gupta, "Fiber Optic Sensors: Principles and Applications", 2006, New India Publications, New Delhi. 